Research Paper

\title{
Serum Osteocalcin Is Significantly Related to Indices of Obesity and Lipid Profile in Malaysian Men
}

\author{
Kok-Yong Chin ${ }^{1}$, Soelaiman Ima-Nirwana ${ }^{\circledR}$, Isa Naina Mohamed ${ }^{1}$, Fairus Ahmad ${ }^{2}$, Elvy Suhana Mohd \\ Ramli², Amilia Aminuddin³, Wan Zurinah Wan Ngah ${ }^{4}$ \\ 1. Pharmacology Department, Faculty of Medicine, Universiti Kebangsaan Malaysia, Kuala Lumpur Malaysia. \\ 2. Anatomy Department, Faculty of Medicine, Universiti Kebangsaan Malaysia, Kuala Lumpur Malaysia. \\ 3. Physiology Department, Faculty of Medicine, Universiti Kebangsaan Malaysia, Kuala Lumpur Malaysia. \\ 4. Biochemistry Department, Faculty of Medicine, Universiti Kebangsaan Malaysia, Kuala Lumpur Malaysia.
}

$\triangle$ Corresponding author: Professor Dr. Soelaiman Ima Nirwana, M.B.B.S., PhD., Department of Pharmacology, Faculty of Medicine, Universiti Kebangsaan Malaysia, Jalan Raja Muda Abdul Aziz, 50300 Kuala Lumpur, Malaysia. Tel: 03-40405514; Fax: 03-26938205; Email: imasoel@ppukm.ukm.edu.my

(C) Ivyspring International Publisher. This is an open-access article distributed under the terms of the Creative Commons License (http://creativecommons.org/ licenses/by-nc-nd/3.0/). Reproduction is permitted for personal, noncommercial use, provided that the article is in whole, unmodified, and properly cited.

Received: 2013.07.12; Accepted: 2013.08.3I; Published: 2014.01.05

\begin{abstract}
Background and aim: Recent studies revealed a possible reciprocal relationship between the skeletal system and obesity and lipid metabolism, mediated by osteocalcin, an osteoblast-specific protein. This study aimed to validate the relationship between serum osteocalcin and indices of obesity and lipid parameters in a group of Malaysian men.

Methods: A total of 373 men from the Malaysian Aging Male Study were included in the analysis. Data on subjects' demography, body mass index (BMI), body fat (BF) mass, waist circumference (WC), serum osteocalcin and fasting lipid levels were collected. Bioelectrical impendence (BIA) method was used to estimate BF. Multiple linear and binary logistic regression analyses were performed to analyze the association between serum osteocalcin and the aforementioned variables, with adjustment for age, ethnicity and BMI.

Results: Multiple regression results indicated that weight, BMI, BF mass, BF \%, WC were significantly and negatively associated with serum osteocalcin $(p<0.00 \mathrm{I})$. There was a significant positive association between serum osteocalcin and high density lipoprotein $(H D L)$ cholesterol $(p=0.032)$. Binary logistic results indicated that subjects with low serum osteocalcin level were more likely to be associated with high BMI (obese and overweight), high BF\%, high WC and low HDL cholesterol $(p<0.05)$. Subjects with high osteocalcin level also demonstrated high total cholesterol level $(p<$ 0.05 ) but this association was probably driven by high HDL level. These variables were not associated with serum C-terminal of telopeptide crosslinks in the subjects $(p>0.05)$.

Conclusion: Serum osteocalcin is associated with indices of obesity and HDL level in men. These relationships should be validated by a longitudinal study, with comprehensive hormone profile testing.
\end{abstract}

Key words: Body mass index; Cholesterol; Men; Obesity; Osteocalcin.

\section{Introduction}

It is an established fact that high body mass index (BMI) is protective of osteoporosis (1). An increase in BMI is often associated with improvement in bone health, as assessed by bone mineral density and calcaneal quantitative ultrasonography $(2,3)$. This is attributed to the ability of our skeletal system to adapt to changes in mechanical loading (4). Bone mass is also influenced by body fat via leptin, a hormone secreted by white adipocytes (5). Systemic infusion of leptin was shown to increase bone mass of rats, but 
intracerebroventricular infusion exerted negative effects on bone mass of rats $(6,7)$. Hence, it is clear that our body composition exerted regulatory control on bone health by mechanical loading (via BMI) and leptin (via body fat).

Since our body operates on the principle of homeostasis, it is reasonable to consider that bone will also exert a reciprocal regulation on body composition. Recently, this was demonstrated by two separate in vivo studies, and the key mediator involved was shown to be osteocalcin, a bone matrix protein synthesized by osteoblast $(8,9)$. Osteocalcin is a marker of bone formation, and it is used together with bone resorption markers to assess bone turnover (10). Mutant mice lacking the gene coding osteocalcin, were shown to be abnormally fat, have high fat pad mass, higher triglyceride level and were glucose intolerant (9). Infusion of osteocalcin in wild type mice was also proven to prevent obesity caused by high fat diet and gold thioglucose-induced hyperphagia (8).

The association between osteocalcin and fat mass and lipid parameters in humans was first demonstrated by Kindblom et al. in Osteoporotic Fractures in Men Study (MrOS) conducted on the Swedish male population (11). This was followed by subsequent reports on other populations, such as the Korean (12), the Chinese (13) and the American populations (14). The majority of the studies agreed that osteocalcin level was associated with obesity and fat mass, but the findings on lipid parameters were less clear $(11,13$, 15). For example, HDL cholesterol was found to be negatively (15), positively (11) or not associated at all (13) with osteocalcin level in different populations.

The aim of this study was to provide evidence that osteocalcin was associated with obesity and lipid profile in Malaysian men, whereby such relationship had not been examined in this population previously. We hope that the information generated would enhance the knowledge on the newly discovered regulation of the skeletal system on body composition mediated by osteocalcin because the number of human studies available is still limited.

\section{Materials and Methods}

This study was conducted as part of the Malaysian Aging Male Study, which aimed to assess the various physiological changes of Malaysian men, particularly the Malays and the Chinese, across a broad age range $(16,17)$. The recruitment was performed from September 2009 to September 2011. A total of 840 men aged 20 years and above volunteered for this study, of which 373 men had serum for osteocalcin and C-terminal telopeptide collagen crosslinks (CTX) assays. The subjects answered a questionnaire on their demographic details and health status. The age of the subjects was determined by the information on their identification cards. The ethnicity of the subjects was declared by themselves. Qualified physicians performed history taking and basic physical examination on the subjects. Subjects with pre-existing bone anomalies, such as osteoporosis, osteomalacia, Paget's disease etc. were excluded from the study. Subjects taking medication known to affect bone health status and bone remodelling were also excluded. Individuals with mobility problems, fractured or underwent major surgery within six months of the screening day were not included in the current study. The inclusion and exclusion criteria were clearly stated in the invitation to the participants. All subjects were informed of the details of the study and their written consent was obtained before they enrolled in the study. The protocol of the study was reviewed and approved by the Research and Ethics Committee of Universiti Kebangsaan Malaysia Medical Centre.

The height of the subjects was measured using a portable stadiometer (SECA, Hamburg, Germany) and was recorded to the nearest $0.1 \mathrm{~cm}$. The body weight, fat mass and body fat percentage of the subjects were determined using a bioelectrical impedance device, BC-418 Segmental Body Composition Analyzer (TANITA Corp, Tokyo, Japan). Briefly, the resistance in which electrical current passed through the body was used to infer the amount of fat in the body. A constant current source $(50 \mathrm{kHz}, 500 \mu \mathrm{A})$ was passed through the body of the subject via electrodes on the tips of the toes of both feet and fingertips of both hands to achieve this. The voltage was measured on the heel of both feet and thenar side of both hands. The weight and fat mass were recorded to the nearest $0.1 \mathrm{~kg}$ and the body fat percentage to the nearest 0.1 $\%$. The short term in vivo precision of the body composition analyzer used was $1.11 \%$ with respect to body fat measurement. Body mass index (BMI) was calculated using the conventional formula: BMI $\left(\mathrm{kg} / \mathrm{m}^{2}\right)=$ body weight of subject $(\mathrm{kg}) /$ squared height of the subject $\left(\mathrm{m}^{2}\right)$. The waist circumference (WC) of the subjects was measured using a soft measuring tape midway between the lowest rib margin and the iliac crest in the standing position. It was recorded to the nearest $1 \mathrm{~cm}$.

The blood of the subjects was collected between 0830 and 1030 after an overnight fast of at least eight hours. Serum was extracted immediately after the blood collection. Part of the serum was sent immediately to an accredited laboratory for evaluation of lipid profile, while the remaining serum was stored at $-70^{\circ} \mathrm{C}$ until analysed. Lipid profile (total cholesterol, triglycerides and HDL cholesterol) was determined using ADVIA Centaur analyzer (Siemens Healthcare 
Diagnostics, Illinois, USA). The LDL cholesterol level was calculated using Friedewald's equation. Serum osteocalcin and CTX were measured using enzyme-linked-immunoassay kits (IDS, Boldon, Tyne and Wear, UK). The inter-assay coefficient of variation of total cholesterol, triglycerides, HDL, osteocalcin and CTX were 0.48-0.86 \%, 0.53-1.02 \%, 0.56-0.79\%, 2.7-5.1 \% and 2.5-10.9\%.

\section{Data analysis}

Data analysis was performed using Statistical Package for Social Sciences version 16 (SPSS Inc., Chicago, USA). The normality of the data was assessed using Kolmogorov-Smirnov test. Lipid parameters and bone remodelling markers did not assume a normal distribution so they were log-transformed and then analysed. The differences of the studied variables between the Malays and the Chinese were compared using independent t-test (for normal data) and Mann-Whitney U-test (for skewed data). The correlation between the variables was explored using Spearman's correlation test. The linear association between osteocalcin and indices of obesity and lipid profile was analysed using multiple linear regression, with adjustment for confounding variables such as age, ethnicity and BMI. Subjects were also dichotomised according to variables studied, into normal/high BMI (overweight and obese), body fat percentage, waist circumference, TG, TC, LDL and HDL groups. The cut-off values for the divisions aforementioned were $\geq 25 \mathrm{~kg} / \mathrm{m}^{2}$ for high BMI, $>25 \%$ for high body fat, $\geq 90 \mathrm{~cm}$ for high waist circumference, $\geq 5.2 \mathrm{mmol} / 1$ for high TC, $\geq 1.7 \mathrm{mmol} / 1$ for high $\mathrm{TG}, \geq 2.6 \mathrm{mmol} / 1$ for high LDL, $\leq 1.0 \mathrm{mmol} / 1$ for low HDL $(18,19)$. A binary logistic regression analysis was performed based on these classifications to assess the relationship between osteocalcin level with obesity and lipid status of the subjects. P-values $<0.05$ were considered statistically significant for all statistical analyses.

\section{Results}

The subjects consisted of 373 men, with mean age 45.35 years (ranged 20 to 79 years), of which 36.5 $\%$ was Malays and $63.5 \%$ was Chinese. There were no significant differences $(p>0.05)$ between Malays and Chinese in their age, body anthropometric parameters (except height) and lipid profiles. Significant differences in serum osteocalcin $(\mathrm{p}<0.001)$ and CTX $(\mathrm{p}<$ 0.001 ) were observed between the two ethnic groups. The levels of both the bone remodeling markers were significantly higher $(\mathrm{p}<0.001)$ in the Malays compared to the Chinese (Table 1). This indicated that the following regression analyses should be adjusted for ethnicity.

A correlation analysis was performed to assess the associations among the studied variables. It was revealed that age and BMI were significantly correlated $(p<0.05)$ to most of the variables of interest (Table 2). Therefore, the following analyses were also adjusted for age and BMI.

Multiple linear regression analysis was conducted to determine the association between osteocalcin with indices of obesity and lipid variables. It showed that body weight $(\beta=-0.237, p<0.001)$, BMI $(\beta=-0.235, p<0.001)$, fat mass $(\beta=-0.235, p<0.001)$ and percentage of body fat $(\beta=-0.180, p<0.001)$ were inversely and significantly associated with serum osteocalcin level after adjustment for age and ethnicity. There was also a positive and significant association between HDL cholesterol and serum osteocalcin $(\beta=$ $0.119, \mathrm{p}=0.032)$. However, the significance of these associations diminished ( $p>0.05)$ after adjustment for BMI was performed (Table 3 ). This suggested that the association between serum osteocalcin and these variables were not independent of BMI.

Binary logistic regression analysis was performed to study the association of serum osteocalcin and the phenotype of obesity and lipid status. The results indicated that subjects with high serum osteocalcin level was significantly less likely to be associated with being overweight or obese $[\mathrm{OR}=0.128$ (0.039-0.417), $\mathrm{p}=0.001]$, having high body fat percentage [OR $=0.206(0.063-0.676), p=0.009$ ] and having central obesity (as shown by having high waist circumference) [OR $=0.244(0.076-0.781), p=0.018]$. A higher serum osteocalcin level was also associated with lower odds of having a low HDL cholesterol level [OR $=0.134(0.029-0.627), p=0.011]$. However, subjects with high serum osteocalcin level were also significantly more likely to be associated with having high total cholesterol level [OR = 3.396 (1.043-11.059), $\mathrm{p}=0.042]$. This was probably due to a significant increase of HDL cholesterol associated with increased osteocalcin level. All these associations were adjusted for age and ethnicity. Further adjustment for BMI reverted these associations to become not significant ( $p>0.05$ ), except for high total cholesterol, again showing that these associations were not independent of BMI (Table 4).

We repeated the logistic regression analysis by substituting serum osteocalcin level with serum CTX level. None of the variables were significantly associated with serum CTX, with or without adjustment for BMI $(p>0.05)$ (Table 5). 
Table I: Characteristics of the subjects.

\begin{tabular}{|c|c|c|c|c|}
\hline & Ethnicity & & & \\
\hline Variable & Malay $(n=136)$ & Chinese $(n=237)$ & Overall $(n=373)$ & p-valuet \\
\hline Age (years) & $45.35(15.36)$ & $46.97(13.52)$ & $46.38(14.22)$ & 0.320 \\
\hline Weight (kg) & $68.69(11.96)$ & 69.36(10.93) & 69.11(11.31) & 0.581 \\
\hline Height $(\mathrm{cm})$ & $165.95(5.87)$ & $168.62(6.00)$ & $167.65(6.08)$ & $<0.001$ \\
\hline Body mass index $\left(\mathrm{kg} / \mathrm{m}^{2}\right)$ & $24.89(2.93)$ & $24.38(3.48)$ & $24.57(3.65)$ & 0.191 \\
\hline Fat mass $(\mathrm{kg})$ & $17.39(8.39)$ & $16.09(6.04)$ & $16.56(6.99)$ & 0.423 \\
\hline Body fat percentage (\%) & $22.64(6.03)$ & $22.52(5.41)$ & $22.57(5.64)$ & 0.498 \\
\hline Waist circumference $(\mathrm{cm})$ & $89.02(10.03)$ & $88.46(8.66)$ & $88.67(9.18)$ & 0.587 \\
\hline Serum osteocalcin* $(\mathrm{ng} / \mathrm{ml})$ & $19.71(8.60)$ & $14.37(6.73)$ & $16.32(7.89)$ & $<0.001$ \\
\hline Serum $\mathrm{CTX}^{*}(\mathrm{ng} / \mathrm{ml})$ & $0.78(0.42)$ & $0.43(0.21)$ & $0.56(0.35)$ & $<0.001$ \\
\hline Triglycerides* $(\mathrm{mmol} / \mathrm{l})$ & $1.57(0.83)$ & $1.46(0.74)$ & $1.50(0.77)$ & 0.257 \\
\hline Total cholesterol* $(\mathrm{mmol} / \mathrm{l})$ & $5.61(1.04)$ & $5.48(0.96)$ & $5.53(0.99)$ & 0.247 \\
\hline $\mathrm{LDL}^{*}(\mathrm{mmol} / \mathrm{l})$ & $3.67(0.96)$ & $3.52(0.81)$ & $3.58(8.71)$ & 0.270 \\
\hline $\mathrm{HDL}^{*}(\mathrm{mmol} / \mathrm{l})$ & $1.26(0.27)$ & $1.28(0.29)$ & $1.27(0.29)$ & 0.638 \\
\hline
\end{tabular}

All values are expressed in mean (standard deviation)

$\mathrm{tp}$-value for the comparison of the variables studied between Malay and Chinese subjects. P-value in bold indicates statistical significance.

*transformed data is used for analysis.

Abbreviation: $\mathrm{CTX}=\mathrm{C}$-terminal telopeptide collagen crosslinks; HDL=high density lipoprotein cholesterol; LDL=low density lipoprotein cholesterol.

Table 2. Correlation analysis between age and BMI with the variables of interest.

\begin{tabular}{lll}
\hline Variables & Age & BMI \\
\hline Age & - & - \\
BMI & $0.147(0.004)$ & - \\
Waist Circum. & $0.250(<0.001)$ & $0.869(<0.001)$ \\
Body Fat $\%$ & $0.209(<0.001)$ & $0.891(<0.001)$ \\
Osteoclacin & $-0.162(0.002)$ & $-0.170(0.001)$ \\
CTX & $-0.129(0.012)$ & $-0.087(0.092)$ \\
Total Cholesterol & $0.276(<0.001)$ & $0.133(0.010)$ \\
Triglycerides & $0.198(<0.001)$ & $0.393(<0.001)$ \\
HDL & $0.103(0.047)$ & $-0.339(<0.001)$ \\
LDL & $0.207(<0.001)$ & $0.145(0.005)$
\end{tabular}

Spearman's correlation is used for the analysis. Significant correlations are printed in bold.

Abbreviation: $\mathrm{CTX}=\mathrm{C}$-terminal telopeptide collagen crosslinks; HDL=high density lipoprotein cholesterol; LDL=low density lipoprotein cholesterol.

Table 3. Linear regression results on the association between the variables studied and serum osteocalcin in the whole study population.

\begin{tabular}{|c|c|c|c|c|}
\hline Overall & Model 1 & & Model 2 & \\
\hline Variable & $\beta$-coefficient & p-value & $\beta$-coefficient & p-value \\
\hline Weight & -0.237 & $<0.001$ & $\mathrm{n} / \mathrm{a}$ & $\mathrm{n} / \mathrm{a}$ \\
\hline BMI & -0.235 & $<0.001$ & $\mathrm{n} / \mathrm{a}$ & $\mathrm{n} / \mathrm{a}$ \\
\hline Body fat mass & -0.235 & $<0.001$ & -0.058 & 0.099 \\
\hline Body fat $\%$ & -0.215 & $<0.001$ & -0.008 & 0.744 \\
\hline WC & -0.180 & $<0.001$ & -0.027 & 0.296 \\
\hline TG & -0.076 & 0.166 & 0.009 & 0.866 \\
\hline TC & 0.049 & 0.356 & 0.073 & 0.182 \\
\hline HDL & 0.119 & 0.032 & 0.024 & 0.642 \\
\hline LDL & 0.055 & 0.318 & 0.083 & 0.135 \\
\hline
\end{tabular}

Model 1 is adjusted for age and ethnicity. Model 2 is adjusted for age, ethnicity and body mass index. P-value printed in bold indicates significant associations.

Abbreviation: $\mathrm{BMI}=$ body mass index; $\mathrm{HDL}=$ high density lipoprotein cholesterol; $\mathrm{LDL}=$ low density lipoprotein cholesterol; $\mathrm{TC}=$ total cholesterol; $\mathrm{TG}=$ triglyceride; $\mathrm{WC}=$ waist circumference.

Table 4. Binary logistic regression results on the association between osteocalcin and obesity and lipid status in the studied population.

\begin{tabular}{llll}
\hline Overall & Model 1 & & Model 2 \\
\hline & OR (95\% CI) & p-value & OR (95\% CI) \\
Overweight/Obese & $\mathbf{0 . 1 2 8}(\mathbf{0 . 0 3 9 - 0 . 4 1 7 )}$ & $\mathbf{0 . 0 0 1}$ & $\mathrm{n} / \mathrm{a}$-value \\
High BF\% & $\mathbf{0 . 2 0 6 ( 0 . 0 6 3 - 0 . 6 7 6 )}$ & $\mathbf{0 . 0 0 9}$ & $0.826(0.147-4.634)$ \\
High WC & $\mathbf{0 . 2 4 4 ( 0 . 0 7 6 - 0 . 7 8 1 )}$ & $\mathbf{0 . 0 1 8}$ & $1.582(0.281-8.899)$ \\
High TG & $0.357(0.110-1.161)$ & 0.087 & $0.581(0.171-1.978)$ \\
High TC & $\mathbf{3 . 3 9 6 ( 1 . 0 4 3 - 1 1 . 0 5 9 )}$ & $\mathbf{0 . 0 4 2}$ & $\mathbf{4 . 2 9 7 ( 1 . 2 7 2 - 1 4 . 5 2 3 )}$ \\
Low HDL & $\mathbf{0 . 1 3 4 ( 0 . 0 2 9 - 0 . 6 2 7 )}$ & $\mathbf{0 . 0 1 1}$ & $0.241(0.048-1.196)$ \\
High LDL & $4.182(0.651-26.858)$ & 0.132 & $5.821(0.824-41.092)$
\end{tabular}

Model 1 is adjusted for age and ethnicity. Model 2 is adjusted for age, ethnicity and body mass index. P-value printed in bold indicates significant associations.

Abbreviation: $\mathrm{BF} \%=$ body fat percentage; $\mathrm{CI}=$ confidence interval; $\mathrm{HDL}=$ high density lipoprotein cholesterol; $\mathrm{LDL}=\mathrm{low}$ density lipoprotein cholesterol; $\mathrm{OR}=\mathrm{odd}$ ratio;

$\mathrm{TC}=$ total cholesterol; $\mathrm{TG}=$ triglyceride; $\mathrm{WC}=$ waist circumference. 
Table 5. Binary logistic regression results on the association between CTX and obesity and lipid status in the studied population.

\begin{tabular}{llll}
\hline Overall & Model 1 & & Model 2 \\
\hline & OR $(95 \%$ CI & p-value & OR (95\% CI) \\
Overweight/Obese & $0.541(0.204-1.436)$ & 0.217 & n/a \\
High BF\% & $0.433(0.157-1.195)$ & 0.106 & $0.543(0.123-2.400)$ \\
High WC & $0.446(0.165-1.208)$ & 0.112 & $0.832(0.189-3.659)$ \\
High TG & $0.419(0.150-1.171)$ & 0.097 & $0.513(0.180-1.464)$ \\
High TC & $2.445(0.880-6.796)$ & 0.086 & $2.679(0.952-7.541)$ \\
Low HDL & $0.847(0.262-2.737)$ & 0.782 & $1.116(0.335-3.714)$ \\
High LDL & $4.077(0.825-20.147)$ & 0.085 & $4.646(0.916-23.565)$ \\
\hline
\end{tabular}

Model 1 is adjusted for age and ethnicity. Model 2 is adjusted for age, ethnicity and body mass index.

Abbreviation: $\mathrm{BF} \%=$ body fat percentage; $\mathrm{CI}=$ confidence interval; $\mathrm{HDL}=$ high density lipoprotein cholesterol; $\mathrm{LDL}=\mathrm{low}$ density lipoprotein cholesterol; $\mathrm{OR}=\mathrm{odds}$ ratio; $\mathrm{TC}=$ total cholesterol; $\mathrm{TG}=$ triglyceride; $\mathrm{WC}=$ waist circumference.

\section{Discussion}

The current study demonstrated that serum osteocalcin level was significantly associated with indices of obesity (BMI, BF and WC) and serum HDL cholesterol level. The odds of subjects with lower serum osteocalcin level being categorized as having high BMI (overweight/obese), high BF percentage, high WC, and low HDL cholesterol were significantly higher. Overall, this would likely predispose subjects with low osteocalcin level to obesity related complications like cardiovascular diseases. There was also a significant association between high serum osteocalcin level and high total cholesterol level. However, it was probably driven by an increase of HDL level rather than LDL level because a high serum osteocalcin level was significantly associated with a high HDL level but not LDL level. We also repeated the logistic regression using CTX-1, a bone resorption marker and found no significant association. This indicated that the association between osteocalcin and metabolic phenotypes was exclusive to osteocalcin alone but not with other bone markers.

The negative association between serum osteocalcin and BMI and body fat was a general finding for most previous studies, regardless of gender, age group and ethnicity. A study performed by Kindblom et al. in Swedish elderly men revealed that plasma osteocalcin level was significantly and negatively associated with weight, BMI, fat mass (assessed using dual-energy X-ray absorptiometry; DEXA) and body fat percentage of the subjects (11). Similarly, Pittas et al. showed that in a group of American elderly men and women, serum osteocalcin was significantly and inversely related to BMI, body fat (using DEXA), and body fat percentage (20). Polgreen et al. also indicated that serum total and carboxylated osteocalcin was significantly and negatively related to BMI and waist circumference in a group of young adults emerging from adolescence (21). However, in these young adult subjects, body fat percentage was not associated with osteocalcin level, suggesting that the influence of os- teocalcin on body fat accumulation might occur later in life (21). Most the study aforementioned used DEXA to measured BF. In view of this aspect, our study was novel by showing that BF estimated using BIA was significantly associated with serum osteocalcin in men.

The relationship between osteocalcin and lipid parameters was an issue of controversy. The current study found that most lipid parameters (TG and LDL cholesterol), excluding HDL, were not related to serum osteocalcin level. A non-significant relationship between serum osteocalcin and LDL cholesterol was recorded by Kindblom et al. (11) and Lee et al. (22) in a population of Korean post-menopausal women. Tan et al. observed that in Mainland Chinese men (age range from young adults to elderly), with each quartile decline in serum osteocalcin level, the likelihood of subjects having low HDL increased (23). Kindblom et al. also found that serum osteocalcin was positively and significantly correlated to HDL, although the effect size of this relationship was small $(\mathrm{r}=0.02)(11)$. However, both Tan et al. and Kindblom et al. also observed a negative and significant relationship between serum osteocalcin and TG levels in their study population $(11,23)$. Furthermore, the study by Saleem et al. indicated that regardless of gender and race, serum osteoclacin level was not significantly correlated to HDL cholesterol but was correlated to TG level in the American population consisting of African Americans and Caucasians (14). While the relationship between serum osteocalcin and TG was negative in the current study, it was not significant. We prudently suggested that an increase in sample size would increase the power of the study, thus making the association become significant. One study by Zhou et al. in a Chinese male population detected a significant negative association between HDL cholesterol and serum osteocalcin level (15). Meanwhile, a study by Bao et al. in another Chinese male population found a significant and positive relationship between serum osteocalcin and LDL cholesterol (13). The sample size employed in the studies of Zhou et al. 
(254 men) and Bao et al. (181 men) were relatively small and this might contribute to the inconsistency of their results compared to the general findings.

There was a paucity of research on how osteocalcin would regulate the level of HDL cholesterol. Previous studies found that osteocalcin was positively associated with adiponectin level $(14,24)$. In some studies, adiponectin was positively related to HDL cholesterol level and was protective of cardiovascular diseases $(25,26)$. Adiponectin level was also associated with a decrease in the catabolic rate of ApoA-I (major apolipoprotein particle of HDL) (27). Based on these observations, we deduced that the positive association between osteocalcin and HDL cholesterol was mediated by adiponectin. However, the detail molecular mechanism of this association had not been established.

On the other hand, the associations between serum osteocalcin and most variables studied were diminished after further adjustment for BMI. This showed that BMI had a strong confounding effect on osteocalcin and studied variables. Another possible explanation could be that the relationship between osteocalcin and the studied variable was mediated by BMI. Hence, we concluded that the association between osteocalcin and metabolic phenotypes was independent of BMI. This was not an isolated finding, as the study of Polgreen et al. also found that the significance of the association between osteocalcin levels and metabolic variables diminished after adjustment for BMI (21).

The possible mechanisms on the regulation of energy metabolism by osteocalcin were illustrated by two remarkable experimental studies. Lee et al. showed that osteocalcin deficient (Ocn-/-) mice were fat, hyperglycemic and insulin-insensitive. Tyrosine phosphatase deficient (Esp-/-) mice originally protected from obesity and hyperglycemia, if lacking one allele of osteocalcin gene, would lose their unusual phenotype. Insulin and adiponectin were the two important hormones in mediating the metabolic phenotype of the mutant mice (9). Ferron et al. treated wild type mice, mice on high fat diet, and mice with drug-induced hyperphagia with recombinant osteocalcin. In all animals, treated mice showed decreased body mass, fat mass, triglyceride level and better regulation of glucose metabolism compared to their untreated counterparts (8). These two studies provided direct evidence of the regulation of osteocalcin on metabolic phenotypes. The results of the current studies further implied that such regulation was possible in humans.

Osteocalcin in carboxylated and undercarboxylated forms could be found in peripheral blood, while total osteocalcin level was more often used in monitoring bone remodelling (28). Previous animal and cell-based studies showed that administration of undercarboxylated osteocalcin was active in the regulation of energy metabolism (8). However, in humans, the evidence was conflicting. While some studies found that undercarboxylated osteocalcin was correlated to energy metabolism, some did not (28). In one recent study in a female population, both carboxylated and undercarboxylated osteocalcin were associated with metabolic syndrome but the direction between the two associations was different (29). This study, as most of the other studies (11, 14, 15, 23), measured the total $\mathrm{OC}$ and did not differentiate the two forms.

Several improvements could be made to enhance the evidence of this study. Glucose metabolism was an important aspect of regulation of energy expenditure and previous studies indicated that osteocalcin was strongly related to glucose metabolism by altering pancreatic beta cell proliferation and insulin sensitivity $(8,9)$. Therefore, insulin, adiponectin and glucose parameters should be included in further studies to illustrate how osteocalcin regulates energy metabolism and subsequently obesity. Body fat percentage was evaluated using bioelectrical impendence (BIA) method rather than DEXA due to logistic difficulties. However, a previous study denoted that body fat assessment using BIA and DEXA showed high correlation (30). The BIA device was not able to differentiate body fat according to body segments. Thus, we were not able to examine the association between individual body fat compartments and osteocalcin level. On the other hand, not many studies used BIA method to examine the relationship between BF and serum osteocalcin level in men. This would be a novel aspect of this study. Due to the cross-sectional nature of this study, causal relationship between osteocalcin and obesity and HDL cholesterol regulation could not be affirmed. A longitudinal study should be conducted to validate this claim. Generalization of the results of this study should be done with caution because we adopted a non-randomized sampling method, and the study population was skewed to the Chinese population in Malaysia.

In conclusion, the current study showed that serum osteocalcin is significantly associated with obesity and HDL cholesterol level in men. This information highlighted that bone is not merely an endocrine target, but it may also exert control on metabolic phenotypes such as obesity and HDL cholesterol level. This study also provides additional information on the complex network of risk factors that contribute to the occurrence of obesity. A longitudinal study should be performed in future to provide an affirmative answer on the relationship among osteocalcin, 
obesity and lipid profile in men.

\section{Acknowledgments}

We thank Universiti Kebangsaan Malaysia for funding this study via grants UKM-AP-TKP-09-2009, FF-376-2010 and DIP-2012-07.

\section{Competing Interests}

The authors have declared that no competing interest exists.

\section{References}

1. Barrera G, Bunout D, Gattás V, de la Maza MP, Leiva L, Hirsch S. A high body mass index protects against femoral neck osteoporosis in healthy elderly subjects. Nutrition. 2004; 20: 769-71.

2. Chin KY, Soelaiman IN, Mohamed IN, Ibrahim S, Wan Ngah WZ. The effects of age, physical activity level, and body anthropometry on calcaneal speed of sound value in men. Arch Osteoporos. 2012; 7: 135-45.

3. Felson DT, Zhang Y, Hannan MT, Anderson JJ. Effects of weight and body mass index on bone mineral density in men and women: The Framingham study. J Bone Miner Res. 1993; 8: 567-73.

4. Wardlaw GM. Putting body weight and osteoporosis into perspective. Am J Clin Nutr. 1996; 63: 433S-6S.

5. Morberg CM, Tetens I, Black E, Toubro S, Soerensen TIA, Pedersen O, et al. Leptin and Bone Mineral Density: A Cross-Sectional Study in Obese and Nonobese Men. J Clin Endocrinol Metab. 2003; 88: 5795-800.

6. Ducy P, Amling M, Takeda S, Priemel M, Schilling AF, Beil FT, et al. Leptin inhibits bone formation through a hypothalamic relay: a central control of bone mass. Cell. 2000; 100: 197-207.

7. Steppan CM, Crawford DT, Chidsey-Frink KL, Ke H, Swick AG. Leptin is a potent stimulator of bone growth in ob/ob mice. Regul Pept. 2000; 92: 73-8.

8. Ferron M, Hinoi E, Karsenty G, Ducy P. Osteocalcin differentially regulates $\beta$ cell and adipocyte gene expression and affects the development of metabolic diseases in wild-type mice. Proc Natl Acad Sci. 2008; 105: 5266-70.

9. Lee NK, Sowa H, Hinoi E, Ferron M, Ahn JD, Confavreux C, et al. Endocrine regulation of energy metabolism by the skeleton. Cell. 2007; 130: 456-69.

10. Seibel MJ. Biochemical markers of bone turnover: part I: biochemistry and variability. Clin Biochem Rev. 2005; 26: 97-122.

11. Kindblom JM, Ohlsson C, Ljunggren Ö, Karlsson MK, Tivesten $\AA$, Smith U, et al. Plasma Osteocalcin Is Inversely Related to Fat Mass and Plasma Glucose in Elderly Swedish Men. J Bone Miner Res. 2009; 24: 785-91.

12. Bae SJ, Choe JW, Chung YE, Kim BJ, Lee SH, Kim HY, et al. The association between serum osteocalcin levels and metabolic syndrome in Koreans. Osteoporos Int. 2011; 22: 2837-46.

13. Bao $Y$, Zhou M, Lu Z Li H, Wang $Y$, Sun $L_{\text {e }}$ et al Serum levels of osteocalcin are inversely associated with the metabolic syndrome and the severity of coronary artery disease in Chinese men. Clin Endocrinol (Oxf). 2011; 75: 196-201.

14. Saleem U, Mosley TH, Kullo IJ. Serum Osteocalcin Is Associated With Measures of Insulin Resistance, Adipokine Levels, and the Presence of Metabolic Syndrome. Arterioscler Thromb Vasc Biol. 2010; 30: 1474-8.

15. Zhou M, Ma X, Li H, Pan X, Tang J, Gao $Y$, et al Serum osteocalcin concentrations in relation to glucose and lipid metabolism in Chinese individuals. Eur J Endocrinol. 2009; 161: 723-9.

16. Chin K-Y, Soelaiman I-N, Mohamed IN, Ngah WZW. Serum testosterone, sex hormone-binding globulin and total calcium levels predict the calcaneal speed of sound in men. Clinics. 2012; 67: 911-6.

17. Chin K-Y, Soelaiman I-N, Naina Mohamed I, Shahar S, Teng NIMF, Suhana Mohd Ramli E, et al. Testosterone is associated with age-related changes in bone health status, muscle strength and body composition in men. Aging Male. 2012; 15: 240-5

18. Alberti KGMM, Eckel RH, Grundy SM, Zimmet PZ Cleeman JI, Donato KA, et al. Harmonizing the Metabolic Syndrome: A Joint Interim Statement of the International Diabetes Federation Task Force on Epidemiology and Prevention; National Heart, Lung, and Blood Institute; American Heart Association; World Heart Federation; International Atherosclerosis Society; and International Association for the Study of Obesity. Circulation. 2009; 120: 1640-5.

19. National Cholesterol Education Program (NCEP) Expert Panel. Third Report of the National Cholesterol Education Program (NCEP) Expert Panel on Detection, Evaluation, and Treatment of High Blood Cholesterol in Adults (Adult Treatment Panel III) Final Report. Circulation. 2002; 106: 3143.

20. Pittas AG, Harris SS, Eliades M, Stark P, Dawson-Hughes B. Association between Serum Osteocalcin and Markers of Metabolic Phenotype. J Clin Endocrinol Metab. 2009; 94: 827-32.

21. Polgreen LE, Jacobs DR, Nathan BM, Steinberger J, Moran A, Sinaiko AR. Association of Osteocalcin With Obesity, Insulin Resistance, and Cardiovascular Risk Factors in Young Adults. Obesity. 2012; 20: 2194-201.
22. Lee SW, Jo HH, Kim MR, You YO, Kim JH. Association between obesity, metabolic risks and serum osteocalcin level in postmenopausal women. Gynecol Endocrinol. 2012; 28: 472-7.

23. Tan A, Gao Y, Yang X, Zhang H, Qin X, Mo L, et al. Low serum osteocalcin level is a potential marker for metabolic syndrome: results from a Chinese male population survey. Metabolism. 2011; 60: 1186-92.

24. Kanazawa I, Yamaguchi T, Yamamoto M, Yamauchi M, Kurioka S, Yano S, et al. Serum Osteocalcin Level Is Associated with Glucose Metabolism and Atherosclerosis Parameters in Type 2 Diabetes Mellitus. J Clin Endocrinol Metab. 2009; 94: 45-9.

25. Meilleur KG, Doumatey A, Huang H, Charles B, Chen G, Zhou J, et al. Circulating Adiponectin Is Associated with Obesity and Serum Lipids in West Africans. J Clin Endocrinol Metab. 2010; 95: 3517-21.

26. Rothenbacher D, Brenner H, März W, Koenig W. Adiponectin, risk of coronary heart disease and correlations with cardiovascular risk markers. Eur Heart J. 2005; 26: 1640-6.

27. Vergès B, Petit JM, Duvillard L, Dautin G, Florentin E, Galland F, et al. Adiponectin Is an Important Determinant of ApoA-I Catabolism. Arterioscler Thromb Vasc Biol. 2006; 26: 1364-9.

28. Patti A, Gennari L, Merlotti D, Dotta F, Nuti R. Endocrine actions of osteocalcin. Int J Endocrinol. 2013; 2013: ID846480.

29. Liu JJ, Toy WC, Wong MD, Tan CS, Tavintharan S, Wong MS, et al. Elevated undercarboxylated and reduced carboxylated osteocalcin are associated with metabolic syndrome in middle age Asian females. Exp Clin Endocrinol Diabetes. 2013; 121: 329-33.

30. Pietrobelli A, Rubiano F, St-Onge MP, Heymsfield SB. New bioimpedance analysis system: improved phenotyping with whole-body analysis. Eur J Clin Nutr. 2004; 58: 1479-84. 